\title{
The First Isomorphism Theorem on QI-algebras
}

\author{
Lee Sassanapitax \\ Department of Mathematics, Faculty of Science, Burapha University \\ Chonburi, Thailand \\ Email: lee.sa [AT] buu.ac.th
}

\begin{abstract}
The aim of this paper is to construct the first isomorphism theorem of QI-homomorphism of QIalgebras. The concepts of normal QI-subalgebras and quotient QI-algebras are also investigated.
\end{abstract}

Keywords- QI-algebra, homomorphism, isomorphism, normal, quotient

\section{INTRODUCTION}

In 1966, the concept of BCK-algebras was introduced by Y. Imai and K. Iseki [4]. Moreover, K. Iseki [5] gave the definition of BCI-algebras in 1980. Both of them play an important role in the study of logical algebras. Afterwards, several structures of algebras such as BH-algebras [6], TM-algebras [7] and KU-algebras [12] were introduced and investigated. The fundamental concepts of abstract algebra such as ideals, congruences and homomorphisms were also studied on those algebraic structures (see [1], [11], [13]). Furthermore, many generalizations of BCK-algebras were introduced by several researchers. Some examples of such algebras are BH-algebras [8], B-algebras [9] and Q-algebras [10]. It turns out that many properties of these kind of algebras were extensively investigated. In 2017, A. B. Saeid, H. S. Kim and A. Razaei proposed a new algebra which is a generalization of implicative BCK-algebras, called a BI-algebra [14]. They provided the basic properties of BI-algebras and discussed about ideals and congruence relations. The properties of ideals of BI-algebras were continuously investigated in [2]. Lately, the notion of QI-algebras, which is a generalization of BI-algebras, was introduced by R. K. Bandaru [3]. The concept of ideals and some basic properties were also considered. One can see more examples of research papers in this area in [15-18].

In this paper, we gave the concept of QI-homomorphisms of QI-algebras and investigated some relate properties. The relations between QI-isomorphisms and quotient QI-algebras are also provided.

\section{PRELIMINARIES}

In this section, we begin with the definition of a QI-algebra which is an algebra $(X, *, 0)$ of type $(2,0)$, i.e., a nonempty set $X$ equipped with a binary operation $*$ and a constant 0 . We also recall some notions and properties of QI-algebras.

Definition 2.1. [3] An algebra $(X, *, 0)$ of type $(2,0)$ is called a QI-algebra if

$$
\begin{aligned}
& \text { (QI1) } x * x=0, \\
& \text { (QI2) } x * 0=x, \\
& \text { (QI3) } x *(y *(x * y))=x * y,
\end{aligned}
$$


for all $x, y \in X$.

The relation " $\leq$ " on a QI-algebra $(X, *, 0)$ is defined by $x \leq y$ if and only if $x * y=0$. From (QI1), we can immediately conclude that $\leq$ is reflexive, however $\leq$ is not a partially ordered relation.

Example 2.2. Let $X=\{0,1,2\}$ be a set with the following Cayley table.

\begin{tabular}{|c|c|c|c|}
\hline$*$ & 0 & 1 & 2 \\
\hline 0 & 0 & 2 & 1 \\
\hline 1 & 1 & 0 & 1 \\
\hline 2 & 2 & 2 & 0 \\
\hline
\end{tabular}

Then, by using computer programming, it is easy to check that $(X, *, 0)$ is a QI-algebra.

Definition 2.3. [3] A QI-algebra $(X, *, 0)$ is said to be right distributive or left distributive, respectively if

$$
(x * y) * z=(x * z) *(y * z) \text { or } z *(x * y)=(z * x) *(z * y),
$$

respectively, for all $x, y, z \in X$.

Example 2.4. Notice that a QI-algebra $(X, *, 0)$ in Example 2.2 is not a right distributive since

$$
(1 * 1) * 1=0 * 1=2 \neq 0=0 * 0=(1 * 1) *(1 * 1),
$$

and $(X, *, 0)$ is not left distributive QI-algebra since

$$
2 *(1 * 0)=2 * 1=2 \neq 0=2 * 2=(2 * 1) *(2 * 0) .
$$

Example 2.5. [3] Let $Y=\{0,1,2,3\}$ be a set with the following Cayley table.

\begin{tabular}{|c|c|c|c|c|}
\hline$*^{\prime}$ & 0 & 1 & 2 & 3 \\
\hline 0 & 0 & 0 & 0 & 0 \\
\hline 1 & 1 & 0 & 1 & 0 \\
\hline 2 & 2 & 2 & 0 & 2 \\
\hline 3 & 3 & 0 & 1 & 0 \\
\hline
\end{tabular}

Then it is easy to check that $\left(Y, *^{\prime}, 0\right)$ is a right distributive QI-algebra.

Proposition 2.6. [3] Let $(X, *, 0)$ be a QI-algebra.

(i) If $X$ is a left distributive QI-algebra, then $X=\{0\}$.

(ii) If $X$ is a right distributive QI-algebra, then $0 * x=0$ for all $x \in X$.

Definition 2.7. [3] Let $(X, *, 0)$ be a QI-algebra and $I$ be a subset of $X$. Then $I$ is called an (QI-)ideal of $X$ if it satisfies the following:

(I1) $0 \in X$,

(I2) for each $x, y \in X$, if $x * y \in I$ and $y \in I$ then $x \in I$.

Example 2.8. [3] Let $X=\{0,1,2\}$ be a set with the following Cayley table.

\begin{tabular}{|c|c|c|c|c|}
\hline$*$ & 0 & 1 & 2 & 3 \\
\hline 0 & 0 & 2 & 1 & 0 \\
\hline 1 & 1 & 0 & 1 & 0 \\
\hline 2 & 2 & 2 & 0 & 2 \\
\hline 3 & 3 & 2 & 1 & 0 \\
\hline
\end{tabular}

Then it is easy to check that $(X, *, 0)$ is a QI-algebra. Note that $I_{1}=\{0,1\}$ and $I_{2}=\{0,1,3\}$ are ideals but $I_{3}=\{0,1,2\}$ is not an ideal of $X$. 


\section{MAIN RESULTS}

In this section, we give the definition of normal QI-subalgebra, congruence relation and QI-homomorphism of QI-algebra. Note that such definitions were provided analogue to the definitions on BI-algebras given in [2]. The first isomorphism theorem on QI-algebras is proven at the end of this section.

Definition 3.1. Let $(X, *, 0)$ be a QI-algebra. A nonempty subset $S$ of $X$ is called a QI-subalgebra of $X$ if it is closed under the operation $*$, i.e., $x * y \in S$ for any $x, y \in S$.

Note that every QI-subalgebra contains 0 since it is nonempty and the axiom (QI1).

Definition 3.2. Let $(X, *, 0)$ be a QI-algebra. A nonempty subset $N$ of $X$ is called a normal subset of $X$ if for each $x, y, a, b \in X, x * y, a * b \in N$ implies $(x * a) *(y * b) \in N$.

Proposition 3.3. Let $N$ be a normal subset of a QI-algebra $(X, *, 0)$. Then $N$ is a QI-subalgebra of $X$.

Proof. Assume that $N$ is a normal subset of $X$. Let $x, y \in N$. Then $x * 0=x \in N$ and $y * 0=y \in N$. Since $N$ is normal subset of $X$, it follows that $x * y=(x * y) *(0 * 0) \in N$. Hence $N$ is closed under $*$. Thus $N$ is a QIsubalgebra of $X$.

From the above proposition, we will call a normal subset of a QI-algebra $(X, *, 0)$ a normal QI-subalgebra $X$. In general, the converse of Proposition 3.3 does not hold as it was shown in the following examples.

Example 3.4. Let $X=\{0,1,2,3\}$ be a set with the following Cayley table.

\begin{tabular}{|c|c|c|c|c|}
\hline$*$ & 0 & 1 & 2 & 3 \\
\hline 0 & 0 & 0 & 0 & 0 \\
\hline 1 & 1 & 0 & 0 & 0 \\
\hline 2 & 2 & 0 & 0 & 2 \\
\hline 3 & 3 & 0 & 3 & 0 \\
\hline
\end{tabular}

Then, by using computer programming, it is easy to check that $(X, *, 0)$ is a QI-algebra. Notice that $A=\{0,1,2\}$ is a QIsubalgebra of $X$ but it is not normal since $3 * 3=0 \in A, 2 * 3=2 \in A$ but $(3 * 2) *(3 * 3)=3 * 0=3 \notin A$.

Example 3.5. Let $X=\{0,1,2,3\}$ be a set with the following Cayley table.

\begin{tabular}{|c|c|c|c|c|}
\hline$*$ & 0 & 1 & 2 & 3 \\
\hline 0 & 0 & 0 & 0 & 0 \\
\hline 1 & 1 & 0 & 1 & 1 \\
\hline 2 & 2 & 2 & 0 & 2 \\
\hline 3 & 3 & 3 & 3 & 0 \\
\hline
\end{tabular}

Then, by using computer programming, it is easy to check that $(X, *, 0)$ is a QI-algebra and $N=\{0,1\}$ is normal. Moreover, we have that $M=\{0,1,2\}$ is a QI-subalgebra and QI-ideal of $X$. Since $3 * 3=0 \in M, 2 * 3=2 \in M$ and $(3 * 2) *(3 * 3)=3 * 0=3 \notin M$, we have that $M$ is not normal. 
Lemma 3.6. Let $N$ be a normal QI-subalgebra of a QI-algebra $(X, *, 0)$ and $x, y \in N$. If $x * y \in N$, then $y * x \in N$.

Proof. Assume that $x * y \in N$. Since $N$ is QI-subalgebra of $X$, it follows that $y * y=0 \in N$. The fact that $y * y, x * y \in N$ and $N$ is normal implies that $y * x=(y * x) * 0=(y * x) *(y * y) \in N$.

Definition 3.7. Let $N$ be a normal QI-subalgebra of a QI-algebra $(X, *, 0)$. A relation $\sim_{N}$ is defined by for each $x, y \in X$,

$$
x \sim_{N} y \text { if and only if } x * y \in N .
$$

Proposition 3.8. Let $N$ be a normal QI-subalgebra of a QI-algebra $(X, *, 0)$. Then $\sim_{N}$ is a congruence relation on $X$.

Proof. Let $x, y, z, w \in X$. Since $x * x=0 \in N$, we have that $x \sim_{N} x$. This means that $\sim_{N}$ is reflexive. From Lemma 3.6, it follows that $x \sim_{N} y$ implies $y \sim_{N} x$. Thus $\sim_{N}$ is symmetric. To show that $\sim_{N}$ is transitive, assume that $x \sim_{N} y$ and $y \sim_{N} z$. Then $x * y, y * z \in N$. Since $\sim_{N}$ is symmetric, $z * y \in N$. This implies that

$$
x * z=(x * z) * 0=(x * z) *(y * y) \in N
$$

because $x * y, z * y \in N$ and $N$ is normal. Therefore, $\sim_{N}$ is an equivalence relation on $X$.

Next, we will show that $\sim_{N}$ is a congruence relation on $X$. Assume that $x \sim_{N} y$ and $z \sim_{N} w$. Then $x * y, y * z \in N$. Since $N$ is normal, $(x * z) *(y * w) \in N$. That is $x * z \sim_{N} y * w$, as required.

Definition 3.9. Let $N$ be a normal QI-subalgebra of a QI-algebra $(X, *, 0)$ and $x \in X$. A congruence class $[x]_{N}$ of $X$ is denoted to be the set $\left\{y \in X: x \sim_{N} y\right\}$. Define $X / N$ to be the set of all congruence class of $X$. That is

$$
X / N=\left\{[x]_{N}: x \in X\right\}
$$

The proof of the following lemma is straightforward, we omit the proof.

Lemma 3.10. Let $N$ be a normal QI-subalgebra of a QI-algebra $(X, *, 0)$ and $x, y \in X$. Then

$$
[x]_{N}=[y]_{N} \text { if and only if } x \sim_{N} y
$$

Theorem 3.11. Let $N$ be a normal QI-subalgebra of a QI-algebra $(X, *, 0)$. Then the binary operation $*^{\prime}$ on $X / N$ defined by

$$
[x]_{N} *^{\prime}[y]_{N}=[x * y]_{N},
$$

for all $x, y \in X$, makes $\left(X / N, *^{\prime},[0]_{N}\right)$ into a QI-algebra. Moreover, $[0]_{N}=N$.

Proof. First, we will show that $*^{\prime}$ is well-defined. Let $x_{1}, y_{1}, x_{2}, y_{2} \in X$ such that $\left[x_{1}\right]_{N}=\left[x_{2}\right]_{N}$ and $\left[y_{1}\right]_{N}=\left[y_{2}\right]_{N}$. 
Then $x_{1} \sim_{N} x_{2}$ and $y_{1} \sim_{N} y_{2}$. Since $\sim_{N}$ is a congruence relation, $x_{1} * y_{1} \sim_{N} x_{2} * y_{2}$. From Lemma 3.10 , it can be concluded that $\left[x_{1} * y_{1}\right]_{N}=\left[x_{2} * y_{2}\right]_{N}$, i.e., $\left[x_{1}\right]_{N} *^{\prime}\left[y_{1}\right]_{N}=\left[x_{2}\right]_{N} *^{\prime}\left[y_{2}\right]_{N}$, as required.

Next, we will show that the axioms of QI-algebra are satisfied. Let $x, y \in X$.

(QI1) $[x]_{N} *^{\prime}[x]_{N}=[x * x]_{N}=[0]_{N}$,

(QI2) $[x]_{N} *^{\prime}[0]_{N}=[x * 0]_{N}=[x]_{N}$,

(QI3) $[x]_{N} *^{\prime}\left([y]_{N} *^{\prime}\left([x]_{N} *^{\prime}[y]_{N}\right)\right)=[x *(y *(x * y))]_{N}=[x * y]_{N}=[x]_{N} *^{\prime}[y]_{N}$.

Moreover, $[0]_{N}=\left\{x \in X: x \sim_{N} 0\right\}=\{x \in X: x * 0 \in N\}=\{x \in X: x \in N\}=N$.

The QI-algebra $X / N$ discussed in the above theorem is called the quotient QI-algebra of $X$ by $N$. Note that the normality of $N$ is required in order to show that $\sim_{N}$ is a congruence relation which implies that $X / N$ is a QI-algebra. follows.

In order to state the isomorphism theorem, the definition of homomorphism in QI-algebra was provided as

Definition 3.12. Let $\left(X, *, 0_{X}\right)$ and $\left(Y, \square, 0_{Y}\right)$ be QI-algebras. A $Q I$-homomorphism is a mapping $f: X \rightarrow Y$ satisfying

$$
f(x * y)=f(x) \sqcup f(y),
$$

for all $x, y \in X$. An injective QI-homomorphism is called QI-monomorphism, a surjective QI-homomorphism is called QI-epimorphism. A QI-isomorphism is a QI-homomorphism which is bijective. We write $X \cong Y$ if there exists a QIisomorphism $f: X \rightarrow Y$.

The kernel of the QI-homomorphism $f$, denoted by $\operatorname{ker} f$, is the set of elements of $X$ that map to $0_{Y}$.

Proposition 3.13. Let $N$ be a normal QI-subalgebra of a QI-algebra $(X, *, 0)$. Then the mapping $\pi: X \rightarrow X / N$ given by

$$
\pi(x)=[x]_{N},
$$

for all $x \in X$, is a QI-epimorphism and $\operatorname{ker} \pi=N$.

Proof. Let $x, y \in X$. Then

$$
\pi(x * y)=[x * y]_{N}=[x]_{N} *^{\prime}[y]_{N}=\pi(x) *^{\prime} \pi(y) .
$$

Hence $\pi$ is a QI-homomorphism. Since

$$
\pi(X)=\{\pi(x): x \in X\}=\left\{[x]_{N}: x \in N\right\}=X / N,
$$

$\pi$ is a QI-epimorphism.

The mapping $\pi$ in the above proposition is called the canonical homomorphism of $X$ onto $X / N$.

Proposition 3.14. Let $\left(X, *, 0_{X}\right),\left(Y, \square, 0_{Y}\right)$ be QI-algebras and $f: X \rightarrow Y$ be a QI-homomorphism and $A \subseteq X$. Then 
(i) $f\left(0_{X}\right)=0_{Y}$.

(ii) If $f$ is a QI-monomorphism, then $\operatorname{ker} f=\left\{0_{x}\right\}$.

(iii) $\operatorname{ker} f$ is a QI-subalgebra of $X$.

(iv) If $A$ is a QI-subalgebra of $X$, then $f(A)$ is a QI-subalgebra of $Y$.

Proof. (i) $f\left(0_{X}\right)=f\left(0_{X} * 0_{X}\right)=f\left(0_{X}\right) \square f\left(0_{X}\right)=0_{Y}$.

(ii) Assume that $f$ is a QI-monomorphism. It follows from (i) that $0_{X} \in \operatorname{ker} f$. To show the converse inclusion, let $x \in \operatorname{ker} f$. Then $f(x)=0_{Y}=f\left(0_{X}\right)$. Since $f$ is injective, $x=0_{X}$. Hence $\operatorname{ker} f=\left\{0_{X}\right\}$.

(iii) Let $x, y \in \operatorname{ker} f$. Then $f(x)=0_{Y}=f(y)$. Thus $f(x * y)=f(x) \square f(y)=0_{Y} \square 0_{Y}=0_{Y}$. Hence $x * y \in \operatorname{ker} f$.

(iv) Suppose that $A$ is a QI-subalgebra of $X$. Let $x, y \in f(A)$. Then $x=f(a)$ and $y=f(b)$ for some $a, b \in A$.

Since $A$ is a QI-subalgebra, $x \square y=f(a) \square f(b)=f(a * b) \in f(A)$. Hence $f(A)$ is a QI-subalgebra of $Y$.

The following example shows that $\operatorname{ker} f$ is not normal, in general.

Example 3.15. Consider a QI-algebra in Example 3.4. Define a mapping $f: X \rightarrow X$ by $f(x)=x$ for all $x \in X$. Then $f$ is a QI-homomorphism and $\operatorname{ker} f=\{0\}$, which is a QI-subalgebra of $X$ but not normal since $2 * 1=0,3 * 1=0$ and $(2 * 3) *(1 * 1)=2 * 0=2 \notin \operatorname{ker} f$.

Definition 3.16. A QI-algebra $(X, *, 0)$ is said to be a $Q I_{1}$-algebra if for each $x, y \in X$,

$$
x * y=0=y * x \text { implies } x=y .
$$

Example 3.17. Let $X=\{0,1,2,3\}$ be a set with the following Cayley table.

\begin{tabular}{|c|c|c|c|c|}
\hline$*$ & 0 & 1 & 2 & 3 \\
\hline 0 & 0 & 0 & 0 & 0 \\
\hline 1 & 1 & 0 & 1 & 2 \\
\hline 2 & 2 & 2 & 0 & 2 \\
\hline 3 & 3 & 3 & 3 & 0 \\
\hline
\end{tabular}

Then, by using computer programming, it is easy to check that $(X, *, 0)$ is a $\mathrm{QI}_{1}$-algebra.

Proposition 3.18. Let $\left(X, *, 0_{X}\right)$ be a $\mathrm{QI}_{1}$-algebra, $\left(Y, \square, 0_{Y}\right)$ a QI-algebra and $\phi: X \rightarrow Y$ a QI-homomorphism. Then $\phi$ is QI-monomorphism if and only if $\operatorname{ker} f=\left\{0_{X}\right\}$.

Proof. The necessity part is Proposition 3.14 (ii). To prove the sufficiency part, assume that $\operatorname{ker} f=\left\{0_{x}\right\}$. Let $x, y \in X$ such that $\phi(x)=\phi(y)$. Then $\phi(x * y)=\phi(x) \square \phi(y)=\phi(x) \square \phi(x)=0_{Y}$. That is $x * y \in \operatorname{ker} f$. Similarly, we 
can show that $y * x \in \operatorname{ker} f$. Since $X$ is a $\mathrm{QI}_{1}$-algebra, $x=y$. Hence $\phi$ is injective.

Proposition 3.19. Let $M$ and $N$ be normal QI-subalgebras of a QI-algebra $(X, *, 0)$ such that $N \subseteq M$. Then $M / N$ is a normal QI-subalgebra of $X / N$.

Proof. Let $\left[x_{1}\right]_{N} *^{\prime}\left[x_{2}\right]_{N},\left[y_{1}\right]_{N} *^{\prime}\left[y_{2}\right]_{N} \in M / N$. Then $\left[x_{1} * x_{2}\right]_{N},\left[y_{1} * y_{2}\right]_{N} \in M / N$. That is $x_{1} * x_{2}, y_{1} * y_{2} \in M$. Since $M$ is normal, $\left(x_{1} * x_{2}\right) *\left(y_{1} * y_{2}\right),\left(x_{1} * y_{1}\right) *\left(x_{2} * y_{2}\right) \in M$. Thus $\left[\left(x_{1} * x_{2}\right) *\left(y_{1} * y_{2}\right)\right]_{N},\left[\left(x_{1} * y_{1}\right) *\left(x_{2} * y_{2}\right)\right]_{N} \in M / N$. Hence $\left(\left[x_{1}\right]_{N} *^{\prime}\left[x_{2}\right]_{N}\right) *^{\prime}\left(\left[y_{1}\right]_{N} *^{\prime}\left[y_{2}\right]_{N}\right),\left(\left[x_{1}\right]_{N} *^{\prime}\left[y_{1}\right]_{N}\right) *^{\prime}\left(\left[x_{2}\right]_{N} *^{\prime}\left[y_{2}\right]_{N}\right) \in M / N$. Therefore, $M / N$ is a normal QIsubalgebra of $X / N$.

In Example 3.5, we have shown that a QI-ideal need not be normal.

Definition 3.20. Let $I$ be a QI-ideal of a QI-algebra $(X, *, 0)$. Then $X$ is called a normal QI-ideal of $X$ if it is normal.

Example 3.21. Let $X=\{0,1,2,3\}$ be a set with the following Cayley table.

\begin{tabular}{|c|c|c|c|c|}
\hline$*$ & 0 & 1 & 2 & 3 \\
\hline 0 & 0 & 2 & 1 & 0 \\
\hline 1 & 1 & 0 & 1 & 1 \\
\hline 2 & 2 & 2 & 0 & 2 \\
\hline 3 & 3 & 2 & 3 & 0 \\
\hline
\end{tabular}

Then, by using computer programming, it is easy to check that $(X, *, 0)$ is a QI-algebra and $I=\{0,3\}$ is a normal QIideal.

Proposition 3.22. Let $(X, *, 0)$ be a QI-algebra and $I \subseteq X$. Then $I$ is a normal QI-subalgebra of $X$ if and only if $I$ is a normal QI-ideal of $X$.

Proof. The sufficiency part follows from Proposition 3.3. To prove the necessity part, let $x, y \in X$ such that $x * y \in I$ and $y \in I$. Since $I$ is a QI-subalgebra, $0 \in I$. Since $0, y \in I$ and $I$ is a QI-subalgebra, we have that $0 * y \in I$. Since $I$ is normal, $x=x * 0=(x * 0) * 0=(x * 0) *(y * y) \in I$. Therefore, $I$ is a QI-ideal of $X$.

Proposition 3.23. Let $\left(X, *, 0_{X}\right),\left(Y, \square, 0_{Y}\right)$ be QI-algebras and $f: X \rightarrow Y$ be a QI-homomorphism. Then ker $f$ is a QI-ideal of $X$.

Proof. Since $f\left(0_{X}\right)=0_{Y}$, we have that $0_{X} \in \operatorname{ker} f$. Let $x, y \in X$ such that $x * y \in \operatorname{ker} f$ and $y \in \operatorname{ker} f$. Then $f(x)=f(x) \square 0_{Y}=f(x) \square f(y)=f(x * y)=0_{Y}$. Thus $x \in \operatorname{ker} f$. Hence $\operatorname{ker} f$ is a QI-ideal of $X$. 
In Example 3.15, we have shown that a kernel of a QI-homomorphism need not be normal.

Definition 3.24. Let $\left(X, *, 0_{X}\right),\left(Y, \square, 0_{Y}\right)$ be QI-algebras and $f: X \rightarrow Y$ be a QI-homomorphism. We say that $f$ is a normal QI-homomorphism if $\operatorname{ker} f$ is a normal QI-ideal of $X$.

Theorem 3.25. (The first isomorphism theorem on QI-algebras) Let $\left(X, *, 0_{X}\right)$ and $\left(Y, \square, 0_{Y}\right)$ be $\mathrm{QI}_{1}$-algebras. If $\varphi: X \rightarrow Y$ be a normal QI-homomorphism, then

$$
X / \operatorname{ker} \varphi \cong \varphi(X)
$$

Proof. Since $\varphi$ is a normal QI-homomorphism, $\operatorname{ker} \varphi$ is normal. Then $X / \operatorname{ker} \varphi$ is a quotient QI-algebra of $X$ by $\operatorname{ker} \varphi$. Let $K=\operatorname{ker} \varphi$. Define a mapping $\phi: X / K \rightarrow Y$ by

$$
\phi\left([x]_{K}\right)=\varphi(x)
$$

for all $x \in X$. We will show that $\phi$ is well-defined. Let $[x]_{K}=[y]_{K} \in X / K$. Then $x \sim_{K} y$. It follows that $x * y, y * x \in K$. Thus $\varphi(x) \square \varphi(y)=\varphi(x * y)=0=\varphi(y * x)=\varphi(y) \square \varphi(x)$. Since $Y \quad$ is $\mathrm{QI}_{1}$-algebra, $\varphi(x)=\varphi(y)$.

That is $\phi\left([x]_{K}\right)=\phi\left([y]_{K}\right)$. Since $\phi\left([x]_{K} *^{\prime}[y]_{K}\right)=\phi\left([x * y]_{K}\right)=\varphi(x * y)=\varphi(x) \square \varphi(y)=\phi\left([x]_{K}\right) \square \phi\left([y]_{K}\right)$, we have that $\phi$ is QI-homomorphism. Next, we will prove that $\phi$ is injective. Clearly, $[0]_{K} \in \operatorname{ker} \phi$. Let $[x]_{K} \in \operatorname{ker} \phi$. Then $\varphi(x)=\phi\left([x]_{K}\right)=0_{Y}$. Thus $x * 0=x \in K$. That is $x \sim_{K} 0_{X}$. It follows that $[x]_{K}=\left[0_{X}\right]_{K}$. Hence $\operatorname{ker} \phi=\left\{[0]_{K}\right\}$. It implies by Proposition 3.18 that $\phi$ is QI- monomorphism. Therefore, $X / K \cong \varphi(X)$.

\section{ACKNOWLEDGMENTS}

The author would like to express his sincere thanks to the referees for their constructive suggestions and valuable comments which improved the standard of this paper.

\section{REFERENCES}

[1] Abdalhussein M. S., "On a Fuzzy Completely Closed Filter with Respect of Element in a BH-algebra" Asian Journal of Applied Science, vol. 5, no. 2, pp. 467-476, 2017.

[2] Ahn S. S., Ko J. M., Saeid A. B., "On Ideals of BI-algebras”, J. Indones. Math. Soc., vol. 25, no. 1, pp. 24-34, 2019.

[3] Bandaru R. K., "On QI-algebras”, Discuss. Math. Gen. Algebra Appl., vol. 37, pp. 137-145, 2017.

[4] Imai Y., Iseki K., "On Axiom Systems of Propositional Calculi”, XIV, Proc, Japan Acad., vol. 42, pp. 19-22, 1966.

[5] Iseki K., "On BCI-algebras", Math. Sem. Notes, Kobe Univ., vol. 8, pp. 125-130, 1980.

[6] Jun Y. B., Roh E. H., Kim H. S., "On BH-algebras”. Scientiae Mathematicae, vol. 1, no. 3, pp. 347-354, 1998.

[7] Megalai M., Tamilarasi A., "TM-algebra-An Introduction", Computer Aided Soft Computing Techniques for Imaging and Biomedical Applications, CASCT, pp. 17-23, 2010.

[8] Neggers J., Ahn S. S., Kim H. S., "On Q-algebras”, Int. J. Math. And Math. Sci., vol 27, pp. 749-757, 2001.

[9] Neggers J., Kim H. S., "On B-algebras", Mate. Vesnik, vol 54, pp. 21-29, 2002.

[10] Neggers J., Kim H. S., “On d-algebras”, Mathematica Slovaca, vol 49, pp. 19-26, 1999.

[11] Prabpayak C., "Some Homomorphism Properties of TM-algebras", Applied Mechanics and Material, vol. 866, pp. 406-409, 2017.

[12] Prabpayak C., Leerawat U., "On Ideal and Conguences in KU-algebras”, Sci.Magna, vol. 5, no. 1, pp. 54-57, 2009

[13] Prabpayak C., Leerawat U., “On Isomorphisms of KU-algebras”, Sci.Magna, vol. 5, no. 3, pp. 25-31, 2009 
[14] Saeid A. B., Kim H. S., Razaei A., “On BI-algebras” An. St. Univ. Ovidius Constanta, vol. 25, pp. 177-194, 2017.

[15] Saeid A. B., Fatemidokht H., Flaut C., Rafsanjani M.K., "Some connections between BCK-algebras and n-ary block codes", Soft Comput., vol. 22, pp. 41-46, 2018.

[16] Saeid A. B., Flaut C., Mayerova S. K., Afshar M., Rafsanjani M.K., “On codes based on BCK-algebras”, J. Intell. Fuzzy Syst., vol. 29, no. 5, pp. 2133-2137, 2015.

[17] Saeid A. B., Motamed S., “A new filter in BL-algebras”, J. Intell. Fuzzy Syst., vol. 27, no. 6, pp. 2949-2957, 2014.

[18] Saeid A. B., Zahiri S., "Radicals in MTL-algebras”, Fuzzy Set Syst., vol. 236, pp. 91-103, 2014. 